\title{
Optical performance of neodymium nanoparticles doped tellurite glasses
}

\begin{abstract}
A series of neodymium NPs doped tellurite glass with composition of (TeO2)0.7(B2O3)0.3]0.7 $(\mathrm{ZnO}) 0.3\} 1-\mathrm{x}(\mathrm{Nd} 2 \mathrm{O} 3 \mathrm{NPs}) \mathrm{x}(\mathrm{x}=0.005,0.01,0.02,0.03,0.04$ and $0.05 \mathrm{~mol} \%)$ was fabricated via melt-quenching method. Density and molar volume were measured and analyzed for the glass series. The optical properties of neodymium NPs doped tellurite glass were measured by $\mathrm{UV}-\mathrm{V}$ is spectrometer, photoluminescence and Z-scan technique. The optical band gap energy of the glass network was in the range of $3.178-3.209 \mathrm{eV}$. The upconversion emission of the laser glass excited at $800 \mathrm{~nm}$ was found in the ultraviolet region. Electronic polarizability, oxide ion polarizability, optical basicity and metallization criterion were calculated. Moreover, linear absorption coefficient, $\alpha$, nonlinear refractive index $\eta(\mathrm{cm} 2 / \mathrm{W})$, nonlinear absorption, $\beta$ (x 10-3) and third order susceptibilities, $\chi($ x 10-6) were measured. The optical efficiency of neodymium NPs doped tellurite glass exhibited excellent properties for its application in laser glass.
\end{abstract}

Keyword: Glass; Tellurite; Neodymium; Nanoparticles; Optical properties 